\title{
TAMING AND THE POINCARÉ CONJECTURE
}

\author{
BY
}

\section{T. L. THICKSTUN}

\begin{abstract}
L. Glaser and L. Siebenmann have shown that the double suspension of a homotopy 3-sphere is homeomorphic to the 5-sphere. This result, together with a well-known characterization of $S^{3}$ due to R. H. Bing, is used to establish a relationship between the Poincare conjecture and two conjectures concerned with taming embeddings in higher dimensions. One of the two conjectures, each of which implies the Poincare conjecture, states, in effect, that a codimension two sphere is tame if it is tame "modulo" a tame disk contained in it.
\end{abstract}

Introduction. In this paper a relationship between the 3-dimensional Poincaré conjecture and two higher dimensional taming conjectures is established. The principal tools, other than standard P.L. topology, are the following two theorems:

Theorem (Glaser [5], Siebenmann [11]). If $M^{3}$ is a homotopy 3-sphere (3-ball) then the double suspension of $M^{3}$ is homeomorphic to $S^{5}\left(B^{5}\right)$.

THEOREM (BING [2]). If $M^{3}$ is a closed 3-manifold in which every embedded circle can be engulfed then $M^{3}$ is homeomorphic to $S^{5}$.

Definitions necessary for the understanding of these theorems as well as those stated in $\$ 1$ are found in $\$ 2$. Throughout this paper the maps and homeomorphisms referred to are not necessarily P.L. unless stated to be. Regular neighborhood theory for polyhedra is used repeatedly. References are [1] and [4].

I would like to take this opportunity to express my gratitude to the referees, who made numerous helpful suggestions as well as corrections in the original version of this paper. In particular, the organization of the proof of Theorem 3 as well as part of the proof itself are due to one of the referees.

\section{Statement of results.}

THEOREM 1. Let $M^{3}$ be a homology 3-sphere and $K$ a subpolyhedron

Received by the editors November 22, 1974 and, in revised form, September 16, 1975 and October 5, 1976.

AMS (MOS) subject classifications (1970). Primary 55A40, 57A10, 57A35; Secondary 57C15, 57C40, 57A45.

Key words and phrases. Suspension, taming, Poincaré conjecture, homology sphere, Heegaard splitting.

- American Mathematical Society 1978 
homeomorphic to a wedge of circles. If there exists a degree one split map $\omega$ : $\left(\Sigma^{n} M^{3}, \Sigma^{n} K\right) \rightarrow S^{n+3}$ for some $n$, such that $\left.\omega\right|_{\Sigma^{n} K}$ is a P.L. homeomorphism onto its image, and if

$$
\left(\left.\omega\right|_{\Sigma^{n} M^{3}-\Sigma^{n} K}\right)_{\#}: \pi_{1}\left(\Sigma^{n} M^{3}-\Sigma^{n} K\right) \rightarrow \pi_{1}\left(S^{n+3}-\omega\left(\Sigma^{n} K\right)\right)
$$

is a monomorphism, then $K$ can be engulfed in $M^{3}$.

COROLlary 1. Let $M^{3}$ be a homology 3-sphere, $K$ a subpolyhedron homeomorphic to a wedge of circles, and $\omega: \Sigma^{n} M^{3} \rightarrow S^{n+3}$ a homeomorphism. If $\left.\omega\right|_{\Sigma^{n} K}$ is tame then $K$ can be engulfed.

THEOREM 2. Let $M^{3}$ be a homology 3-sphere and $K$ a subpolyhedron homeomorphic to a wedge of circles which is a P.L. spine of one of the handlebodies of a Heegaard splitting of $M^{3}$. If there exists a degree one, split map $\omega:\left(\Sigma^{n} M^{3}, \Sigma^{n} K\right) \rightarrow S^{n+3}$ for some $n$, such that $\left.\omega\right|_{\Sigma^{n} K}$ is a P.L. homeomorphism onto its image, then $M^{3}$ is homeomorphic to $S^{3}$.

COROLlaRY 2. Let $M^{3}$ be a homology 3-sphere and $K$ a subpolyhedron homeomorphic to a wedge of circles, which is a P.L. spine of one of the handlebodies of a Heegaard splitting of $M^{3}$. If there exists a degree one split map $\omega:\left(\Sigma^{n} M^{3}, \Sigma^{n} K\right) \rightarrow S^{n+3}$ for some $n$, such that $\left.\omega\right|_{\Sigma^{n} K}$ is tame, then $M^{3}$ is homeomorphic to $S^{3}$.

Note. In the conjectures stated below we refer implicitly to a P.L. structure on $S^{n+3} / e\left(D^{n}\right)$ where $e: D^{n} \rightarrow S^{n+3}$ is a P.L. embedding. This P.L. structure is defined as follows. If $(X, A)$ is a compact polyhedral pair there exists a homeomorphism

$$
h: X / A \rightarrow \overline{X-N(A)} \cup_{\dot{N}(A)} C(\dot{N}(A))
$$

for which $h(A / A)=p$. Here $N(A)$ denotes the P.L. regular neighborhood of $A$ in $X, \dot{N}(A)$ its boundary, $C(\dot{N}(A))$ the cone over $\dot{N}(A)$ and $p$ the cone point. The polyhedral structure of $X / A$ is taken to be the one induced on $X / A$ by this homeomorphism (see [3, Theorem 10.2]). Also note that in the case $(X, A)=\left(S^{n+3}, e\left(D^{n}\right)\right)$ there is a P.L. homeomorphism $h: S^{n+3} / e\left(D^{n}\right)$ $\rightarrow S^{n+3}$ because $e\left(D^{n}\right)$ is ambiently isotopic to $D^{n} \rightarrow S^{n+3}$ (the standard inclusion) by virtue of the codimension.

Conjecture $1_{n}$. Let $\left(S^{n}, D^{n}\right)$ be the standard pair and $e: S^{n+1} \rightarrow S^{n+3}$ an embedding, polyhedral with respect to a possibly noncombinatorial triangulation of $S^{n+3}$, such that $\left.e\right|_{D^{n}}$ is P.L. with respect to the standard triangulation of $S^{n+3}$. Let $h: S^{n+3} / e\left(D^{n}\right) \rightarrow S^{n+3}$ be a P.L. homeomorphism and $e_{*}: S^{n+1} / D^{n} \rightarrow S^{n+3} / e\left(D^{n}\right)$ the embedding induced by $e$. If $h \circ e_{*}$ is tame, then $e$ must be tame.

CONJECTURE $2_{n}$. Let $K$ be a wedge of $k$ circles, $p$ the wedge point, and $e$ : $\left(\Sigma^{n} K, \Sigma^{n} p\right) \rightarrow\left(S^{n+3}, D^{n}\right)$ an embedding with $e\left(\Sigma^{n} p\right)=D^{n}$, polyhedral with 
respect to a possibly noncombinatorial triangulation of $S^{n+3}$. Furthermore, suppose $S^{n+3}-\mathrm{e}\left(\Sigma^{n} K\right)$ is homeomorphic to $\mathbf{R}^{n} \times \dot{H}_{k}$ (where $H_{k}$ is a solid handlebody of genus $k$ ). Let $h: S^{n+3} / e\left(\Sigma^{n} p\right) \rightarrow S^{n+3}$ be a P.L. homeomorphism and $e_{*}: \Sigma^{n} K / \Sigma^{n} p \rightarrow S^{n+3} / e\left(\Sigma^{n} p\right)$ the embedding induced by $e$. If $h \circ e_{*}$ is tame, then $e$ is tame.

THEOREM 3. If Conjecture $1_{n}$ is true for some $n \geqslant 3$, then the 3-dimensional Poincaré conjecture is true.

THEOREM 4. If Conjecture $2_{n}$ is true for some $n \geqslant 3$, then the 3-dimensional Poincaré conjecture is true.

\section{Definitions.}

Definition 1. The nth suspension of a space $X$, denoted by $\Sigma^{n} X$, is defined inductively by $\Sigma^{1} X=X \times[-1,1] \cup_{p}\{-1,1\}$ (sometimes written as $X \times$ $[-1,1] / p)$, where $p: X \times\{-1,1\} \rightarrow\{-1,1\}$ is projection. The suspension points of a single suspension, $\Sigma X$, are -1 and 1 (usually denoted $p_{0}$ and $p_{1}$ ). If $\left\{p_{0}, p_{1}\right\}$ are the suspension points of $\Sigma X$ and $\left\{q_{0}, q_{1}\right\}$ those of $\Sigma Y$, then $f$ : $\Sigma X \rightarrow \Sigma Y$ is a proper map of suspensions if $f^{-1}\left(q_{i}\right)=p_{i}, i=0,1$.

Note that for each $\alpha \in(-1,1)$ we have an injection $i_{\alpha}: L \rightarrow \Sigma L$ defined by $i_{\alpha}(x)=(x, \alpha)$. By abuse of notation we will write $(\Sigma L, L)$ to mean $\left(\Sigma L, i_{0}(L)\right)$. Similarly, if $\left(L_{1}, L_{2}\right)$ is a pair of spaces, we will refer to $L_{1}-L_{2}$ (meaning $i_{0}\left(L_{1}-L_{2}\right)$ ) as a subspace of $\Sigma L_{1}-\Sigma L_{2}$.

Definition 2. A topological embedding $e: L \rightarrow M^{n}$ of a polyhedron into a P.L. manifold is tame if there exists a homeomorphism $h: M \rightarrow M$ such that $h \circ e: L \rightarrow M^{n}$ is polyhedral.

Definition 3. A map $f:(X, A) \rightarrow Y$ is split if $f^{-1}(f(A))=A$ and $f^{-1}(f(X$ $-A))=X-A$. If $X$ is a manifold and $A$ a compact submanifold of codimension 0 , we term $f$ split under the weaker condition $f(X-A) \cap f(A)$ $\subset f(\partial A)$.

Definition 4. A map $f: M^{n} \rightarrow N^{n}$ of compact manifolds with boundary is proper if $f\left(\partial M^{n}\right) \subset \partial N^{n}$ (i.e. $f:\left(M^{n}, \partial M^{n}\right) \rightarrow\left(N^{n}, \partial N^{n}\right)$ is a map of pairs).

Definition 5. A map $f: X \rightarrow Y$ of homology $n$-spheres (not necessarily manifolds) is of degree one if $f_{*}: H_{n}(X) \rightarrow H_{n}(Y)$ is an isomorphism. (Note: The term homology 3-sphere will always refer to a closed 3-manifold.) A map of closed orientable $n$-manifolds, $f: M^{n} \rightarrow N^{n}$, is of degree one if $f_{*}: H_{n}\left(M^{n}\right)$ $\rightarrow H_{n}\left(N^{n}\right)$ is an isomorphism. A proper map of compact orientable $n$-manifolds $f:\left(M^{n}, \partial M^{n}\right) \rightarrow\left(N^{n}, \partial N^{n}\right)$ is of degree one if $f_{*}: H_{n}\left(M^{n}, \partial M^{n}\right) \rightarrow$ $H_{n}\left(N^{n}, \partial N^{n}\right)$ is an isomorphism.

Definition 6. A subset $X$ of a P.L. manifold $M^{n}$ can be engulfed in $M^{n}$ if there exists a subpolyhedron $Q^{n}$ of $M^{n}$ such that $X \subset Q^{n}$ and $Q^{n}$ is a P.L. ball. 


\section{Background lemmas.}

LeMma 1. Let $f:\left(M^{n}, L\right) \rightarrow W^{n}$ be a split map (not necessarily P.L.) of P.L. manifolds with $L$ a compact subpolyhedron of $M^{n}$ and $f(L)$ a subpolyhedron of $W^{n}$. Then $f$ is homotopic to a split map of pairs, $\bar{f}:\left(M^{n}, N_{L}\right) \rightarrow\left(W^{n}, \tilde{N}_{f(L)}\right)$, with $\left.\bar{f}\right|_{N_{L}}$ proper, where $N_{L}$ and $\tilde{N}_{f(L)}$ are regular neighborhoods of $L$ and $f(L)$. Furthermore, if $\left(\left.f\right|_{M^{n}-L}\right)_{\#}: \pi_{1}\left(M^{n}-L\right) \rightarrow \pi_{1}\left(W^{n}-f(L)\right)$ is a monomorphism then $\left(\left.\bar{f}\right|_{M^{n}-N_{L}}\right)_{\#}: \pi_{1}\left(M^{n}-N_{L}\right) \rightarrow \pi_{1}\left(W^{n}-\tilde{N}_{f(L)}\right)$ is also a monomorphism.

Proof. Let $N_{f(L)}$ be a regular neighborhood of $f(L)$. By continuity of $f$ we can choose a regular neighborhood $N_{L}$ of $L$ in $M^{n}$ sufficiently close that $f\left(N_{L}\right) \subset \stackrel{\circ}{N}_{f(L)}$. Also, we have $f\left(N_{L}-L\right) \subset N_{f(L)}-f(L)$ because $f\left(M^{n}-L\right)$ $\subset W^{n}-f(L)$. Now $f\left(\overline{M^{n}-N_{L}}\right)$ does not intersect $f(L)$ and is compact, so we can choose a smaller regular neighborhood $N_{f(L)}^{\prime}$ of $f(L)$ such that $f\left(\overline{M^{n}-N_{L}}\right) \cap N_{f(L)}^{\prime}=\varnothing$ and, therefore, $f\left(\partial N_{L}\right) \subset N_{f(L)}-N_{f(L)}^{\prime}$. By regular neighborhood theory there exists a P.L. homeomorphism $h: N_{f(L)}-\stackrel{\circ}{f(L)}^{\prime}$ $\rightarrow \partial N_{f(L)} \times[-1,1]$, and we can choose this homeomorphism so that

$$
\begin{gathered}
h\left(f\left(\partial N_{L}\right)\right) \subset \partial N_{f(L)} \times\left[-\frac{1}{2}, \frac{1}{2}\right], \\
f\left(N_{L}\right) \subset N_{f(L)}^{\prime} \cup h^{-1}\left(\partial N_{f(L)} \times\left[-1, \frac{1}{2}\right]\right)
\end{gathered}
$$

and $f\left(\overline{M^{n}-N_{L}}\right)$ misses $N_{f(L)}^{\prime} \cup h^{-1}\left(\partial N_{f(L)} \times\left[-1,-\frac{1}{2}\right]\right)$. Let $\varphi:[-1,1] \rightarrow$ $[-1,1]$ be a proper P.L. map such that $\varphi^{-1}(0)=\left[-\frac{1}{2}, \frac{1}{2}\right]$ but $\varphi$ is one-one off of $\left[-\frac{1}{2}, \frac{1}{2}\right]$. The map

$$
h^{-1} \circ(\mathrm{id} \times \varphi) \circ h: N_{f(L)}-\stackrel{\circ}{N}_{f}^{\prime}(L) \rightarrow N_{f(L)} \rightarrow{\stackrel{\circ}{N_{f}^{\prime}}}^{\prime}(L)
$$

is fixed on $\partial\left(N_{f(L)}-\dot{\circ}_{f(L)}^{\prime}\right)=\partial N_{f(L)} \cup \partial N_{f(L)}^{\prime}$ and can therefore be extended to all of $W^{n}$ by making it the identity outside $N_{f(L)}-\dot{N}_{f(L)}^{\prime}$. Call this map $\bar{\varphi}$ : $W^{n} \rightarrow W^{n}$. Then $\bar{f}=\bar{\varphi} \circ f: M^{n} \rightarrow W^{n}$ will be the desired map, where $\tilde{N}_{f(L)}=$ $N_{f(L)}^{\prime} \cup h^{-1}\left(\partial N_{f(L)} \times[-1,0]\right)$. It is easily seen from the construction of $f$ that $\bar{f}$ is homotopic to $f$ by a homotopy $f_{t}$ which is split at each level (i.e. $f_{t}$ sends $M^{n}-L$ into $W^{n}-f(L)$ for all $\left.t\right)$. Therefore, $\left(\left.\bar{f}\right|_{M^{n}-L}\right)_{\#}: \pi_{1}\left(M^{n}-L\right) \rightarrow$ $\pi_{1}\left(W^{n}-f(L)\right)$ is a monomorphism. Also the following diagram clearly commutes:

$$
\begin{array}{ccc}
M^{n}-N_{L} & \stackrel{\bar{f}}{\rightarrow} & W^{n}-\tilde{N}_{f(L)} \\
i_{1} \downarrow & & \downarrow i_{2} \\
M^{n}-L & \stackrel{\bar{f}}{\rightarrow} & W^{n}-f(L)
\end{array}
$$

The vertical arrows are inclusions which, by regular neighborhood theory, are homotopy equivalences. The bottom horizontal map induces a monomorphism on $\pi_{1}$ and, therefore, the top map does also. Q.E.D. 
LEMMA 2. Let $f:\left(M^{3}, U\right) \rightarrow\left(W^{3}, U^{\prime}\right)$ be a split degree one map of homology 3-spheres with $U$ and $U^{\prime}$ handlebodies. Then $\left.f\right|_{\partial u}: \partial U \rightarrow \partial U^{\prime}$ and $\left.f\right|_{u}: U \rightarrow U^{\prime}$ are both degree one maps.

Proof. Let $V$ and $V^{\prime}$ denote the closed complements of $U$ and $U^{\prime}$. By naturality of the Mayer-Vietoris sequence with respect to split maps we get the following commutative diagram:

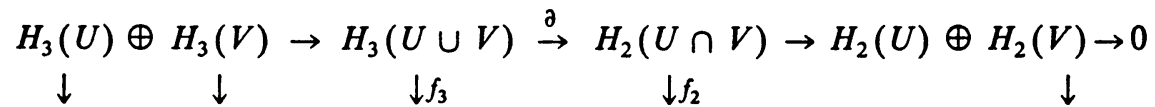

$$
\begin{aligned}
& H_{3}\left(U^{\prime}\right) \oplus H_{3}\left(V^{\prime}\right) \rightarrow H_{3}\left(U^{\prime} \cup V^{\prime}\right) \stackrel{\partial^{\prime}}{\rightarrow} H_{2}\left(U^{\prime} \cap V^{\prime}\right) \rightarrow H_{2}\left(U^{\prime}\right) \oplus H_{2}\left(V^{\prime}\right) \rightarrow 0
\end{aligned}
$$

The vertical maps are induced by $f$. The groups $H_{3}(U), H_{3}\left(U^{\prime}\right), H_{3}(V)$ are all zero $\left(U, U^{\prime}, V, V^{\prime}\right.$ are all compact 3-manifolds with boundary and, therefore, have 2-dimensional spines). Of course, $H_{2}(U)$ and $H_{2}\left(U^{\prime}\right)$ are both 0 , and $f_{3}$ is an isomorphism because $f$ is of degree one. Also $\mathrm{H}_{2}(U \cup V)=\mathrm{H}_{2}\left(U^{\prime} \cup V^{\prime}\right)$ $=0$. First we show that $\partial^{\prime}$ is an isomorphism. If it were not then by the sequence $\mathrm{H}_{2}\left(V^{\prime}\right)$ would necessarily have torsion. By Poincaré duality we have $H_{2}\left(V^{\prime}\right)$ is isomorphic to $H^{1}\left(V^{\prime}, \partial V^{\prime}\right)$ and by the exact sequence of a pair (for cohomology): $H^{1}\left(V^{\prime}\right) \leftarrow H^{1}\left(V^{\prime}, \partial V^{\prime}\right) \leftarrow 0$. So $H^{1}\left(V^{\prime}\right)$ must have torsion if $H_{2}\left(V^{\prime}\right)$ does. But $H^{1}\left(V^{\prime}\right) \cong$ free part $H_{1}\left(V^{\prime}\right) \oplus$ torsion part $H_{0}\left(V^{\prime}\right)$. So $\partial^{\prime}$ must be an isomorphism. Since $\partial^{\prime}$ is an isomorphism, $f_{2} \circ \partial$ must also be an isomorphism, which implies that $f_{2}$ is a surjection. But any surjection of $\mathbf{Z}$ to itself must be an isomorphism so $\left.f\right|_{U \cap V}=\left.f\right|_{\partial U}$ is of degree one.

That $\left.f\right|_{U}: U \rightarrow U^{\prime}$ is of degree one now follows immediately from naturality of the exact sequence of a pair. Explicitly we have

$$
\begin{array}{ccccccc}
0 & \rightarrow & H_{3}(U, \partial U) & \rightarrow & H_{2}(\partial U) & \rightarrow & 0 \\
& & \downarrow & & & \downarrow & \\
0 & \rightarrow & H_{3}\left(U^{\prime}, \partial U^{\prime}\right) & \rightarrow & H_{2}\left(\partial U^{\prime}\right) & \rightarrow & 0
\end{array}
$$

The vertical arrow on the right has been shown to be an isomorphism so the one on the left is also an isomorphism. Q.E.D.

LEMMA 3. Any degree one proper map of a handlebody to a handlebody of the same genus is properly homotopic to a homeomorphism.

Proof. See Lemma 1.1 [12].

LEMMA 4. Let $f: U \rightarrow V^{\prime}$ be a proper map from a handlebody to a compact 3-manifold with boundary which is homeomorphic to the closed complement of a handlebody in $S^{3}$. If $\left.f\right|_{\partial U}$ is a homeomorphism, then $f$ is homotopic to a homeomorphism keeping $\left.f\right|_{\partial U}$ fixed.

Proof. See Hempel [6, Theorem 4]. 
LEMMA 5. Let $X$ and $Y$ be homology $(n-1)$-spheres and $f: \Sigma X \rightarrow \Sigma Y$ be a degree one (in dimension $n$ ) proper map of suspensions. Then the map $(f / \Sigma)_{\alpha}$ : $X \rightarrow Y$, which is obtained by composing the maps $i_{\alpha}: X \rightarrow \Sigma X-\left\{p_{0}, p_{1}\right\}$, $i_{\alpha}(x)=(x, \alpha),\left.f\right|_{\Sigma X-\left\{p_{0}, p_{1}\right\}}: \Sigma X-\left\{p_{0}, p_{1}\right\} \rightarrow \Sigma Y-\left\{q_{0}, q_{1}\right\}=Y \times(-1,1)$ and $\varphi: Y \times(-1,1) \rightarrow Y$, projection, is a degree one map.

Proof. It is easily seen that $(f / \Sigma)_{\alpha}$ is homotopic to $(f / \Sigma)_{\alpha^{\prime}}$ for any $\alpha^{\prime}$, so it is sufficient to demonstrate the lemma for $(f / \Sigma)_{0}$.

First it can be shown that $f$ is homotopic through proper maps to a map $f^{\prime}$ such that

$$
f^{-1}(Y \times[0,1] / Y \times\{1\})=X \times[0,1] / X \times\{1\}
$$

and

$$
f^{\prime-1}(Y \times[-1,0] / Y \times\{-1\})=X \times[-1,0] / X \times\{-1\} .
$$

The proof is left to the reader.

Looking at the Mayer-Vietoris sequence obtained by splitting $\Sigma X$ into $X \times[0,1] / X \times\{1\}$ and $X \times[-1,0] / X \times\{-1\}$ and $\Sigma Y$ similarly, and noting that $f^{\prime}$ respects the splitting, we get the following commutative diagram by naturality of the exact sequence for homology:

$0=H_{n}(X \times[0,1] / X \times\{1\}) \oplus H_{n}(X \times[-1,0] / X \times\{-1\}) \rightarrow H_{n}(\Sigma X) \rightarrow H_{n-1}(X \times\{0\}) \rightarrow 0$
$0=H_{n}(Y \times[0,1] / Y \times\{1\}) \oplus H_{n}(Y \times[-1,0] / Y \times\{-1\}) \rightarrow H_{n}(\Sigma Y) \rightarrow H_{n-1}(Y \times\{0\}) \rightarrow 0$.

The vertical maps are induced by $f^{\prime}$. Since the $f^{\prime}$ is homotopic to $f$, the vertical arrow on the left is an isomorphism, and, hence, the one on the right is also an isomorphism. Q.E.D.

LEMMA 6. Let $\left(L_{1}, L_{2}\right)$ be a P.L. pair, $\varphi: C\left(L_{1}\right) \rightarrow[\varepsilon, 1]$ the P.L. map defined linearly with $\varphi\left(L_{1}\right)=\varepsilon$ and $\varphi(p)=1\left(p\right.$, the cone point, and $L_{1} \subset$ $C\left(L_{1}\right)$ as the base of the cone), and $\psi: K \rightarrow C\left(L_{2}\right)$ a P.L. homeomorphism with $\psi(q)=p$. Also, let $\mathrm{lk} q$ be the link of $q$ in a second derived subdivision of $a$ triangulation of $K$ making $\psi$ simplicial. Then for $\varepsilon^{\prime}$ in $(\varepsilon, 1)$ sufficiently close to 1 there is a P.L. homeomorphism of pairs

$$
h:\left(\varphi^{-1}\left[\varepsilon, \varepsilon^{\prime}\right], \varphi^{-1}\left[\varepsilon, \varepsilon^{\prime}\right] \cap C\left(L_{2}\right)\right) \rightarrow\left[\varepsilon, \varepsilon^{\prime}\right] \times\left(L_{1}, L_{2}\right)
$$

such that $\psi(\mathrm{lk} q) \subset \varphi^{-1}\left[\varepsilon, \varepsilon^{\prime}\right]$ and $h(\psi(\mathrm{lk} q))=\left\{\left(\varepsilon+\varepsilon^{\prime}\right) / 2\right\} \times L_{2}$.

Proof. The proof is an easy application of regular neighborhood theory and is left to the reader.

4. Proofs of Theorems 1 and 2. Both Theorems 1 and 2 are proved by induction on $n$ starting with $n=0$. They parallel each other very closely so we first present the case $n=0$ for Theorem 2 , then the case $n=0$ for Theorem 1. The induction steps are then given in the same order. 
Proof of THeOrem 2 For $n=0$. We have a split map $\omega:\left(M^{3}, K\right) \rightarrow S^{3}$. By Lemma 1, this map $\omega$ is homotopic to a split map (which we continue to call $\omega) \omega:\left(M^{3}, U\right) \rightarrow\left(S^{3}, U^{\prime}\right)$ where (in this case) $U$ is one of the handlebodies in a Heegaard splitting of $M^{3}$. The new map must, of course, also be of degree one, so from Lemma 2 , we can conclude that $\left.\omega\right|_{U}: U \rightarrow U^{\prime}$ is of degree one. By Lemma 3, $\left.\omega\right|_{U}$ is properly homotopic to a homeomorphism $\omega^{\prime}$. Consider the homotopy restricted to $\left.\omega\right|_{\partial U=\partial V}$ (where $V$ is the other handlebody). This homotopy of $\left.\omega\right|_{\partial V}: \partial V \rightarrow \partial V^{\prime}$ (where $V^{\prime}$ is the complement of $\left.\dot{U}^{\prime}\right)$ can be extended to a homotopy of $\left.\omega\right|_{V}: V \rightarrow V^{\prime}$ by the homotopy extension property for polyhedral pairs where the pair is $(V, \partial V) . \omega^{\prime}$ can then be extended to all of $M^{3}$ so that $\omega^{\prime}:\left(M^{3}, U\right) \rightarrow\left(S^{3}, U^{\prime}\right)$ is split with $\left.\omega^{\prime}\right|_{U}$ a homeomorphism. But by Lemma 4 we may replace $\left.\omega^{\prime}\right|_{V}$ with a homeomorphism which is the same as $\omega^{\prime}$ on $\partial V$. We then have a homeomorphism from $M^{3}$ to $S^{3}$. Q.E.D.

Proof OF THEOREM 1 FOR $n=0$. We have a split degree one map $\omega$ : $\left(M^{3}, K\right) \rightarrow\left(S^{3}, L\right)$ with $\left(\left.\omega\right|_{M^{3}-K}\right)_{\#}: \pi_{1}\left(M^{3}-K\right) \rightarrow \pi_{1}\left(S^{3}-L\right)$ a monomorphism. By Lemma 1 we may replace this map by a new split degree one map (still denoted by $\omega) \omega:\left(M^{3}, N_{K}\right) \rightarrow\left(S^{3}, N_{L}\right)$ such that $\left(\left.\omega\right|_{M^{3}-N_{K}}\right)_{\#}$ : $\pi_{1}\left(M^{3}-N_{K}\right) \rightarrow \pi_{1}\left(S^{3}-N_{L}\right)$ is a monomorphism. Applying Lemma 2 and proceeding as in the proof for Theorem 2 we may also ensure that $\left.\omega\right|_{N_{K}}$ : $N_{K} \rightarrow N_{L}$ is a homeomorphism.

Now consider the compact 3-manifold with boundary, $M^{3}-\dot{N}_{K}$. Suppose $Q^{3} \subset M^{3}-N_{K}$ is a compact submanifold with $\partial Q^{3} \simeq S^{2}$. Applying Van Kampen's theorem and the Mayer-Vietoris sequence to the triple $\left(M^{3}, Q^{3}, M^{3}-\dot{Q}^{3}\right)$, it is seen that $Q^{3}$ is 1 -connected and has trivial homology. By the Hurewicz and Whitehead theorems we then conclude that $Q^{3}$ is a homotopy ball (i.e. contractible). By Kneser's decomposition theorem for 3-manifolds [7] we may assume that $M^{3}-\left(\stackrel{\circ}{N}_{K} \cup \dot{Q}^{3}\right)$ is irreducible (i.e. that there is only one homotopy ball in $M^{3}-\dot{N}_{K}$ which is not a standard ball and that it is $Q^{3}$ ). Denote $M^{3}-\dot{N}_{K}$ by $W$ and let $W^{\prime}$ be the irreducible 3-manifold obtained by replacing $Q^{3}$ by a standard ball, $B^{3}$. We certainly can construct a proper homotopy equivalence $q: B^{3} \rightarrow Q^{3}$ such that $\left.q\right|_{\partial B^{3}}$ is a homeomorphism. We may then extend to a map $\bar{q}: W^{\prime} \rightarrow W$ which is a homeomorphism away from $B^{3}$.

Consider the proper map $\omega \circ \bar{q}: W^{\prime} \rightarrow S^{3}-\dot{N}_{L}$. It is a homeomorphism on $\partial W^{\prime}$ and induces a monomorphism on $\pi_{1}$. By the same argument that was applied to $W, S^{3}-\dot{N}_{L}$ must be irreducible if it contains no homotopy balls which are not standard balls. The Schoenflies theorem implies that this is the case so both $W^{\prime}$ and $S^{3}-\dot{N}_{L}$ are irreducible. By Lemma 2 of [9], $\omega \circ \bar{q}$ is homotopic to a homeomorphism (leaving $\partial W^{\prime}$ fixed). Denote this homeomorphism by $(\omega \circ q)^{\prime}$ and the manifold $W^{\prime} \cup_{\partial N_{K}} N_{K}$ by $M^{3^{\prime}}$ (i.e. the 
manifold obtained from $M^{3}$ by replacing $Q^{3}$ with a standard ball). $(\omega \circ \bar{q})^{\prime}$ can, of course, be extended to a split homeomorphism $h:\left(M^{3^{\prime}}, N_{K}\right) \rightarrow$ $\left(S^{3}, N_{L}\right)$. Therefore $K$ can be engulfed in $M^{3^{\prime}}$. Since $K$ does not intersect $B^{3}$, the ball which engulfs $K$ can be chosen to avoid $B^{3}$. Therefore, $K$ can be engulfed in $M^{3}$. Q.E.D.

Proof of the Induction SteP for Theorem 2. The problem is to show that, given a map $\omega_{n}: \Sigma^{n} M^{3} \rightarrow S^{n+3}$, satisfying the given conditions, there exists a map $\omega_{n-1}: \Sigma^{n-1} M^{3} \rightarrow S^{n+2}$ satisfying the corresponding conditions for $n-1$. First we can assume that $\omega_{n}: \Sigma^{1}\left(\Sigma^{n-1} M^{3}\right) \rightarrow \Sigma^{1} S^{n+2}$ is a proper map of suspensions. For if $\left\{q_{0}, q_{1}\right\}$ are the suspension points of $\Sigma^{1} S^{n+2}$ let $h$ : $S^{n+3} \rightarrow S^{n+3}$ be a P.L. homeomorphism sending $\omega_{n}\left(q_{0}\right)$ to $p_{0}$ and $\omega_{n}\left(q_{1}\right)$ to $p_{1}$ (note that since $\left.\omega_{n}\right|_{\Sigma^{n} K}$ is an embedding, $\omega_{n}\left(q_{0}\right) \neq \omega_{n}\left(q_{1}\right)$ ).

Then $\left(h \circ \omega_{n}\right)^{-1}\left(p_{i}\right)=q_{i}$ because $\left(\omega_{n}^{-1} \circ \omega_{n}\right)\left(\Sigma^{n} K\right)=\Sigma^{n} K$. Therefore, $h \circ \omega_{n}: \Sigma^{1}\left(\Sigma^{n-1} M^{3}\right) \rightarrow \Sigma^{1} S^{n+2}$ is a proper map of suspensions. We have $\Sigma^{n} S^{3}=\left(\Sigma^{n-1} S^{3} \times[-1,1]\right) / p$, so we can define a map $\varphi: \Sigma^{n} S^{3} \rightarrow[-1,1]$ by projection onto the second coordinate. Let $\varepsilon$ be the vertex nearest 1 in $[-1,1]$ in a second derived subdivision of $\varphi$. Then $\varphi^{-1}(\varepsilon)$ must be the link of $p_{1}$ in the triangulation of $\Sigma^{n} S^{3}$ and $\varphi^{-1}(\varepsilon) \cap \omega_{n}\left(\Sigma^{n} K\right)$ is the link of $p_{1}$ in the triangulation of $\omega_{n}\left(\Sigma^{n} K\right)$. The star neighborhood of $p_{1}$ is the cone over the link of $p_{1}$ so we have $\varphi^{-1}([\varepsilon, 1])=C\left(\varphi^{-1}(\varepsilon)\right)$ (again $C$ denotes cone). $\varphi^{-1}(\varepsilon)=\Sigma^{n-1} S^{3}$ and, because $\omega_{n}^{-1}\left(p_{1}\right)=q_{1}$, regular neighborhood theory for polyhedra implies that $\varphi^{-1}(\varepsilon) \cap \omega_{n}\left(\Sigma^{n} K\right)$ is P.L. homeomorphic to $\Sigma^{n} K$. From the definition of $\varphi$ it is clear that $\left.\varphi\right|_{\varphi^{-1}(\varepsilon, 1)}: C\left(\Sigma^{n-1} S^{3}\right) \rightarrow[\varepsilon, 1]$ is the linear map obtained by sending $\Sigma^{n-1} S^{3}$ to $\varepsilon$ and $p_{1}$ (the cone point) to 1 . In our application of Lemma $6, q_{1},\left.\varphi\right|_{\varphi^{-1}[\varepsilon, 1]}, \Sigma^{n-1} S^{3}, \varphi^{-1}(\varepsilon) \cap \omega_{n}\left(\Sigma^{n} K\right)$, and $\left.\omega_{n}\right|_{\Sigma^{n} K \cap\left(\omega_{n} \circ \varphi\right)^{-1}(\varepsilon, 1 D}$ will play the roles of $q, \varphi, L_{1}, L_{2}$ and $\psi$, respectively.

Take a triangulation of $\Sigma^{n} M^{3}$ sufficiently fine so that $\omega_{n}\left(\mathrm{lk} q_{1}\right) \subset$ $\varphi^{-1}([\varepsilon, 1])$. Choose $\varepsilon^{\prime}$ sufficiently close to 1 not only for the application of Lemma 6 but so that $\omega_{\mathrm{n}}\left(\mathrm{kk} q_{1}\right) \subset \varphi^{-1}\left(\left[\varepsilon, \varepsilon^{\prime}\right]\right)$. This can be done since $\omega_{n}^{-1}\left(p_{1}\right)$ $=q_{1}$ and $1 \mathrm{k} q_{1}$ is compact. The link of $Q_{1}$ in $\Sigma^{n} K$ is $1 \mathrm{k} q_{1} \cap \Sigma^{n} K$, which will play the role of $\mathrm{lk} q$ in Lemma 6 . Also note that

$$
\begin{aligned}
\varphi^{-1}\left(\left[\varepsilon, \varepsilon^{\prime}\right]\right) & \cap \omega_{n}\left(\Sigma^{n} K\right) \\
= & \varphi^{-1}\left(\left[\varepsilon, \varepsilon^{\prime}\right]\right) \cap C\left(\varphi^{-1}(\varepsilon) \cap \omega_{n}\left(\Sigma^{n} K\right)\right) .
\end{aligned}
$$

From Lemma 6 we then have a P.L. homeomorphism:

$$
\begin{aligned}
h:\left(\varphi^{-1}\left(\left[\varepsilon, \varepsilon^{\prime}\right]\right), \varphi^{-1}\left(\left[\varepsilon, \varepsilon^{\prime}\right]\right) \cap \omega_{n}\left(\Sigma^{n} K\right)\right) \\
\quad \rightarrow\left[\varepsilon, \varepsilon^{\prime}\right] \times\left(\Sigma^{n-1} S^{3}, \varphi^{-1}(\varepsilon) \cap \omega_{n}\left(\Sigma^{n} K\right)\right)
\end{aligned}
$$

with

$$
h\left(\omega_{n}\left(\mathrm{k} q_{1} \cap \Sigma^{n} K\right)\right)=\left\{\left(\varepsilon+\varepsilon^{\prime}\right) / 2\right\} \times\left[\varphi^{-1}(\varepsilon) \cap \omega_{n}\left(\Sigma^{n} K\right)\right]
$$


and, furthermore, $\omega_{n}\left(\mathrm{k} q_{1}\right) \subset \varphi^{-1}\left(\left[\varepsilon, \varepsilon^{\prime}\right]\right)$. Now let

$$
\Phi:\left(\Sigma^{n-1} M^{3}, \Sigma^{n-1} K\right) \rightarrow\left(\mathrm{k} q_{1}, \mathrm{lk} q_{1} \cap \Sigma^{n-1} K\right)
$$

be a P.L. homeomorphism of pairs (which exists by regular neighborhood theory). The desired map will be

$$
\begin{aligned}
\omega_{n-1}=\rho \circ h \circ \omega_{n} \circ \Phi: & \left(\Sigma^{n-1} M^{3}, \Sigma^{n-1} K\right) \\
\rightarrow & \left(\Sigma^{n-1} S^{3}, \varphi^{-1}(\varepsilon) \cap \omega_{n}\left(\Sigma^{n} K\right)\right)
\end{aligned}
$$

where

$$
\begin{aligned}
\rho:\left[\varepsilon, \varepsilon^{\prime}\right] \times[ & \left.\Sigma^{n-1} S^{3}, \varphi^{-1}(\varepsilon) \cap \omega_{n}\left(\Sigma^{n} K\right)\right] \\
\rightarrow & {\left[\Sigma^{n-1} S^{3}, \varphi^{-1}(\varepsilon) \cap \omega_{n}\left(\Sigma^{n} K\right)\right] }
\end{aligned}
$$

is projection. That $\rho \circ h \circ \omega_{n} \circ \Phi$ is of degree one follows from Lemma 5. It is clearly a split map and a P.L. homeomorphism on $\Sigma^{n} K$. Q.E.D.

PROOF OF THE INDUCTION STEP FOR THEOREM 1 . The construction of $\omega_{n-1}$ is the same as for Theorem 2, but, in addition, we must show that if

$$
\left(\left.\omega_{n}\right|_{\Sigma^{n} M^{3}-\Sigma^{n} K}\right)_{\#}: \pi_{1}\left(\Sigma^{n} M^{3}-\Sigma^{n} K\right) \rightarrow \pi_{1}\left(S^{n+3}-\omega_{n}\left(\Sigma^{n} K\right)\right)
$$

is a monomorphism, then

$$
\begin{aligned}
\left(\left.\omega_{n-1}\right|_{\Sigma^{n-1} M^{3}-\Sigma^{n-1} K}\right)_{\#}: & \pi_{1}\left(\Sigma^{n-1} M^{3}-\Sigma^{n-1} K\right) \\
& \rightarrow \pi\left(S^{n+2}-\omega_{n-1}\left(\Sigma^{n-1} K\right)\right)
\end{aligned}
$$

is also a monomorphism.

First note that if $\left(L_{1}, L_{2}\right)$ is a P.L. pair, then $\Sigma L_{1}-\Sigma L_{2}$ is P.L. homeomorphic to $\mathbf{R} \times\left(L_{1}-L_{2}\right)$ and the homeomorphism can be chosen to send $L_{1}-L_{2}$ onto $\{0\} \times\left(L_{1}-L_{2}\right)$. It follows that the inclusion $L_{1}-L_{2} \subset$ $\Sigma L_{1}-\Sigma L_{2}$ is a homotopy equivalence. In particular, the inclusion of $\Sigma^{n-1} M^{3}-\Sigma^{n-1} K$ into $\Sigma^{n} M^{3}-\Sigma^{n} K$ is a homotopy equivalence. By regular neighborhood theory we have a P.L. homeomorphism of triples

$$
H:\left(\Sigma^{n} M^{3}, \text { lk } q_{1}, \text { lk } q_{1} \cap \Sigma^{n} K\right) \rightarrow\left(\Sigma^{n} M^{3}, \Sigma^{n-1} M^{3}, \Sigma^{n-1} K\right) .
$$

Therefore, the inclusion

$$
i: \operatorname{lk} q_{1}-\left(\mathrm{lk} q_{1} \cap \Sigma^{n} K\right) \rightarrow \Sigma^{n} M^{3}-\Sigma^{n} K
$$

is also a homotopy equivalence. Let

$$
j: \varphi^{-1}\left(\left[\varepsilon, \varepsilon^{\prime}\right]\right) \cap\left(S^{n+3}-\omega_{n}\left(\Sigma^{n} K\right)\right) \rightarrow S^{n+3}-\omega_{n}\left(\Sigma^{n} K\right)
$$

be inclusion. We then have a commutative square: 


$$
\begin{aligned}
& \begin{array}{ccc}
\Sigma^{n} M^{3}-\Sigma^{n} K & \left.\omega_{n}\right|_{\Sigma^{n} M^{3}-\Sigma^{n} K} & S^{n+3}-\omega_{n}\left(\Sigma^{n} K\right) \\
i \uparrow & & \uparrow j
\end{array} \\
& \operatorname{lk} q_{1}-\left(\operatorname{lk} q_{1} \cap \Sigma^{n} K\right) \stackrel{\omega_{n} \mid l_{\mathrm{k} q}-\left(\mathrm{lk} q_{1} \cap \Sigma^{n} K\right)}{\rightarrow} \varphi^{-1}\left(\left[\varepsilon, \varepsilon^{\prime}\right]\right) \times\left(S^{n+3}-\omega_{n}\left(\Sigma^{n} K\right)\right) .
\end{aligned}
$$

$i$ is a homotopy equivalence and $\left(\left.\omega_{n}\right|_{\Sigma^{n} M^{3}-\Sigma^{n} K}\right)_{\#}$ a monomorphism by assumption, so it follows that $\left(\left.\omega_{n}\right|_{\Sigma^{n} M^{3}-\Sigma^{n} K}{ }^{\circ} i\right)_{\#}$ is a monomorphism. Therefore, $\left(\left.j \circ \omega_{n}\right|_{1 \mathrm{k} q_{1}-\left(\operatorname{lk} q_{1} \cap \Sigma^{n} K\right)}\right)_{\#}$ is a monomorphism and, hence, $\left(\left.\omega_{n}\right|_{1 \mathrm{k} q_{1}-\left(\mathrm{lk} q_{1} \cap \Sigma^{n} K\right)}\right)_{\#}$ is also a monomorphism. $\left.\omega_{n-1}\right|_{\Sigma^{n-1} M^{3}-\Sigma^{n-1} K}$ is the composition of the following maps:

$$
\begin{aligned}
& \Sigma^{n-1} M^{3}-\Sigma^{n-1} K \stackrel{\Phi}{\rightarrow} \mathrm{lk} q_{1}-\left(\mathrm{lk} q_{1} \cap \Sigma^{n} K\right) \\
& \stackrel{\omega_{n}}{\rightarrow} \varphi^{-1}\left(\left[\varepsilon, \varepsilon^{\prime}\right]\right) \cap\left(S^{n+3}-\omega_{n}\left(\Sigma^{n} K\right)\right) \\
& \stackrel{h}{\rightarrow}\left[\varepsilon, \varepsilon^{\prime}\right] \times\left(\Sigma^{n-1} S^{3}-\left[\varphi^{-1}(\varepsilon) \cap \omega_{n}\left(\Sigma^{n} K\right)\right]\right) \\
& \stackrel{\rho}{\rightarrow} \Sigma^{n-1} S^{3}-\left[\varphi^{-1}(\varepsilon) \cap \omega_{n}\left(\Sigma^{n} K\right)\right] .
\end{aligned}
$$

$\Phi$ and $h$ are homeomorphisms and $\rho$ projection so they induce isomorphisms on $\pi_{1}$. Therefore the composition is a monomorphism. Q.E.D.

Corollaries 1 and 2 are immediate from Theorems 1 and 2 and Definition 2.

\section{Proofs of Theorems 3 and 4.}

Proof of Theorem 3. If $M^{3}$ is a homotopy 3-sphere and $K \subset M^{3}$ a P.L. embedded circle, let $B^{3} \subset M^{3}$ be a P.L. ball intersecting $K$ in a single point $p$ (in $\partial B^{3}$ ). Let $M^{3 \prime}=M^{3}-B^{3}$. Siebenmann [11] states that there exists a homeomorphism $\omega: \Sigma^{2} M^{3 \prime} \rightarrow B^{5}$ (the standard 5-ball). Suspending once we get a homeomorphism (still denoted by $\omega) \omega: \Sigma^{3} M^{3 \prime} \rightarrow B^{6}$. Since $\Sigma^{3}\left(\partial M^{3 \prime}\right)$ is a P.L. 5-sphere, the homeomorphism $\left.\omega\right|_{\Sigma^{3}\left(\partial M^{3}\right)}: \Sigma^{3}\left(\partial M^{3 \prime}\right) \rightarrow \partial B^{6}$ is isotopic to a P.L. homeomorphism (by the stable homeomorphism theorem of Kirby [13]). By the standard collaring trick we can therefore assume $\left.\omega\right|_{\Sigma^{3}\left(\partial M^{3}\right)}$ is a P.L. homeomorphism. By coning $\left.\omega\right|_{\Sigma^{3}\left(\partial M^{3}\right)}$ we can extend it to a P.L. homeomorphism of balls and, therefore, can extend $\omega$ to a homeomorphism $\bar{\omega}: \Sigma^{3} M^{3} \rightarrow S^{6}$ such that $\left.\bar{\omega}\right|_{\Sigma^{3} B^{3}}$ is P.L. In particular, $\bar{\omega}$ is P.L. on $\Sigma^{3} p$ (a 3-disk). By suspending $\bar{\omega}$ we get a homeomorphism (still denoted by $\bar{\omega}$ ) $\bar{\omega}$ : $\Sigma^{n} M^{3} \rightarrow S^{n+3}$ for any $n \geqslant 3$. Furthermore, letting $\alpha:\left(S^{n+3}, \bar{\omega}\left(\Sigma^{n} p\right)\right) \rightarrow$ $\left(S^{n+3}, D^{n}\right)$ be a P.L. homeomorphism of pairs (which exists because the codimension is 3$)$, we have a homeomorphism $\alpha \circ \bar{\omega}:\left(\Sigma^{n} M^{3}, \Sigma^{n} p\right) \rightarrow$ $\left(S^{n+3}, D^{n}\right)$ (where $\left(S^{n+3}, D^{n}\right)$ is the standard pair).

Next we show that $\Sigma^{n} M^{3} / \Sigma^{n} p$ is a P.L. sphere of dimension $n+3$. In order to show that a polyhedron is a P.L. manifold of dimension $n+3$ it is sufficient to show that the boundary of the P.L. regular neighborhood of every point is P.L. homeomorphic to the standard P.L. sphere of dimension $n+2$. It is clear from the definition of suspension that the only point in 
$\Sigma^{n} M^{3} / \Sigma^{n} p$ we need worry about is $\Sigma^{n} p / \Sigma^{n} p$. Let $\dot{N}$ denote the boundary of the P.L. regular neighborhood of $\Sigma^{n} p / \Sigma^{n} p$ in $\Sigma^{n} M / \Sigma^{n} p$. It follows from the definition of $\Sigma^{n} M^{3} / \Sigma^{n} p$ that $\dot{N}$ is P.L. bicollared in $\Sigma^{n} M^{3}-\Sigma^{n} p . \dot{N} \times \mathbf{R}^{1}$ is then a P.L. manifold. A regular neighborhood of $\dot{N}$ in $\dot{N} \times \mathbf{R}^{1}$ is $\dot{N} \times$ $[-1,1]$. Its boundary is $\dot{N} \times\{-1,1\}$ and therefore $\dot{N}$ must be a P.L. manifold. We previously established a homeomorphism of the pairs $\left(\Sigma^{n} M^{3}, \Sigma^{n} p\right)$ and $\left(S^{n+3}, D^{n}\right)$ and therefore a homeomorphism between $\Sigma^{n} M^{3} / \Sigma^{n} p$ and $S^{n+3} / D^{n}$, which is in turn homeomorphic to $S^{n+3}$. Therefore, $\Sigma^{n} M^{3} / \Sigma^{n} p$ is a triangulated manifold homeomorphic to $S^{n+3}$. The link of a vertex in a triangulated manifold has the homotopy type of a sphere [10, p. 124] so $\dot{N}$ is a homotopy sphere and, therefore, by the higher dimensional Poincaré conjecture, a P.L. sphere. Thus, $\Sigma^{n} M^{3} / \Sigma^{n} p$ is a P.L. manifold homeomorphic to $S^{n+3}$, so, by the uniqueness of the P.L. structure on $S^{n+3}$, there is a P.L. homeomorphism $\theta: \Sigma^{n} M^{3} / \Sigma^{n} p \rightarrow S^{n+3}$.

Alternate argument. One can also show $\dot{N}$ is a P.L. sphere in the following way. It is seen by inspection that $\dot{N}$ is the boundary of the regular neighborhood of $\Sigma^{n} p$ in $\Sigma^{n} M^{3}$ and by further inspection that this is $\left(\bar{M}^{3} \times S^{n-1}\right)$ $\cup_{\partial \bar{M}^{3} \times D^{n}}\left(\partial \bar{M}^{3} \times D^{n}\right)$, where $\bar{M}^{3}$ is a homotopy ball obtained from $M^{3}$ by deleting the interior of a standard ball in $M^{3}$. It is easily demonstrated, using Van Kampen's theorem, the Mayer-Vietoris sequence, the Hurewicz theorem and the Whitehead theorem, that the above union is a homotopy sphere and, hence, a standard sphere for $n \geqslant 3$.

In the following diagram, $f_{*}: X / A \rightarrow Y / B$ will denote the map induced from $f:(X, A) \rightarrow(Y, B)$. Also let $e=\left.\bar{\omega}\right|_{\Sigma^{n} K}:\left(\Sigma^{n} K, \Sigma^{n} p\right) \rightarrow\left(\bar{\omega}\left(\Sigma^{n} K\right), \bar{\omega}\left(\Sigma^{n} p\right)\right), r:$ $S^{n+3} / D^{n} \rightarrow S^{n+3}$ be a P.L. homeomorphism and denote $h=r \circ \alpha_{*}$. We then have the diagram:

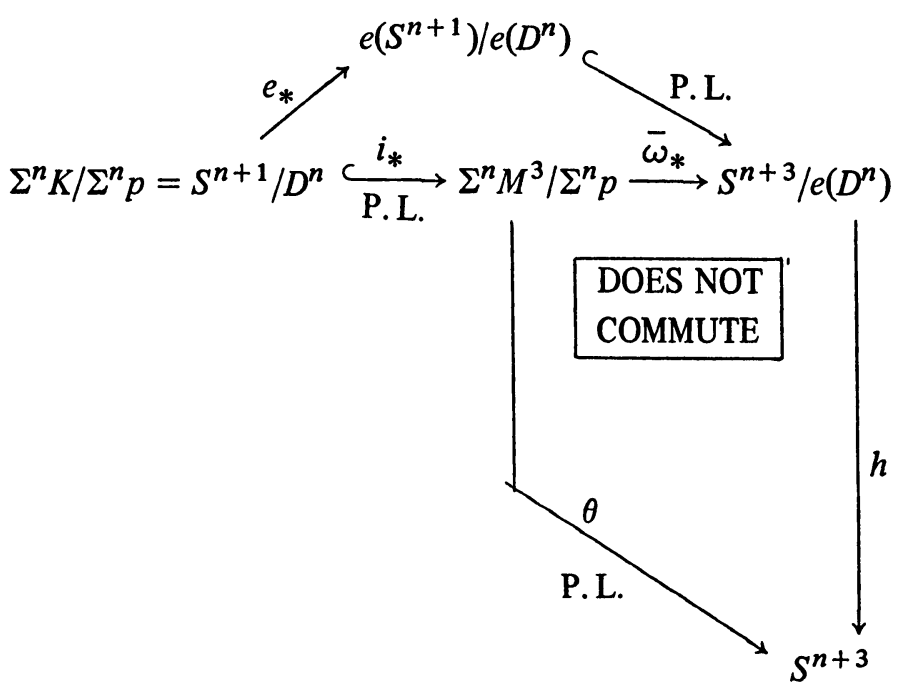


The homeomorphism $\varphi=\theta \circ \bar{\omega}^{-1} \circ h^{-1}$ satisfies $\varphi \circ h \circ e_{*}=\theta \circ i_{*}$ which is a P.L. embedding of $S^{n+1} / D^{n}$ in $S^{n+3}$. Therefore, $h \circ e_{*}$ is tame and, using Conjecture $1_{n}, e$ is tame. Therefore, by Corollary $1, K$, an arbitrary loop in $M^{3}$, can be engulfed and hence, by Bing's characterization of $S^{3}$ [2], $M^{3}$ is the 3-sphere. Q.E.D.

Proof of TheOREM 4. The proof is identical to that of Theorem 3 except that Corollary 2 is used in place of Corollary 1 . In addition, the ball $B^{3}$ in $M^{3}$ is chosen to intersect $e(K)$ at $p$, the wedge point. The condition $S^{n+3}-$ $e\left(\Sigma^{n} K\right)$ homeomorphic to $\mathbf{R}^{n} \times \stackrel{\circ}{H}$ is satisfied if $e: K \rightarrow M^{3}$ is the inclusion of the spine of one of the handlebodies in a Heegaard splitting of $M^{3}$ because $M^{3}-e(K)$ is homeomorphic to $\stackrel{H}{H}$. Q.E.D.

\section{REFERENCES}

1. E. Akin, Manifold phenomena in the theory of polyhedra, Trans. Amer. Math. Soc. 143 (1969), 413-473. MR 40 \#6544.

2. R. H. Bing, Necessary and sufficient conditions that a 3-manifold be $S^{3}$, Ann. of Math. (2) 68 (1958), 17-37. MR 20 \# 1973.

3. M. M. Cohen, Simplicial structures and transverse cellularity, Ann. of Math. (2) 85 (1967), 218-245. MR 35 \# 1037.

4. A general theory of relative regular neighborhoods, Trans. Amer. Math. Soc. 136 (1969), 189-229. MR 40 \#2052.

5. L. C. Glaser, On the double suspension of certain homotopy 3-spheres, Ann. of Math. (2) 85 (1967), 494-507. MR 36 \#886.

6. J. P. Hempel, A surface in $S^{3}$ is tame if it can be deformed into each complementary domain, Trans. Amer. Math. Soc. 111 (1964), 273-287. MR 28 \#3409.

7. H. Kneser, Geschlossen Flächen in drei dimensionalen Mannigfaltigkeiten, Jber. Deutsch. Math. Verein. 38 (1929), 248-260.

8. D. R. McMillan, Jr., Homeomorphisms on a solid torus, Proc. Amer. Math. Soc. 14 (1963), 386-390. MR 27 \# 770.

9. Boundary-preserving mappings of 3-manifolds, Topology of Manifolds, Markham, Chicago, 1970, pp. 161-175. MR 43 \#2723.

10. H. Seifert and W. Threlfall, Lehrbuch der Topologie, New York, 1950.

11. L. C. Siebenmann, Are nontriangulable manifolds triangulable?, Topology of Manifolds, Markham, Chicago, 1970, pp. 77-84. MR 42 \#6837.

12. F. Waldhausen, On mappings of handlebodies and of Heegaard splittings, Topology of Manifolds, Markham, Chicago, 1970, pp. 205-211. MR 42 \#6822.

13. R. C. Kirby, Stable homeomorphisms and the annulus conjecture, Ann. of Math. (2) 89 (1969), 575-582. MR 39 \#3499.

7270 Carrizo Drive, la Jolla, California 92037 